\title{
Verticillin A Inhibits Leiomyosarcoma and Malignant Peripheral Nerve Sheath Tumor Growth via Induction of Apoptosis
}

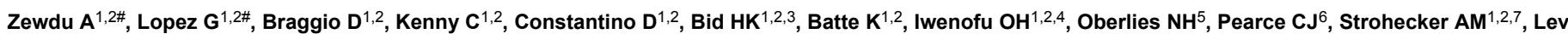
$D^{8}$ and Pollock RE R. $^{*}$

${ }^{1}$ Department of Surgical Oncology, Ohio State University Wexner Medical Center, Columbus, Ohio, USA

${ }^{2}$ The James Cancer Center, Ohio State University Wexner Medical Center, Columbus, Ohio, USA

${ }^{3}$ Resonant Therapeutics, Inc., Ann Arbor, Michigan, USA

${ }^{4}$ Department of Pathology, Comprehensive Cancer Center, Ohio State University, Columbus, Ohio, USA

${ }^{5}$ Department of Chemistry and Biochemistry, University of North Carolina at Greensboro, Greensboro, North Carolina, USA

${ }^{6}$ Mycosynthetix, Inc., Hillsborough, North Carolina, USA

${ }^{7}$ Department of Cancer Biology and Genetics, Ohio State University, Columbus, Ohio, USA

${ }^{8}$ Surgery B, Sheba Medical Center, Tel Aviv, Israel

"Corresponding author: Pollock RE, Department of Surgical Oncology, Comprehensive Cancer Center, Ohio State University, 410 West 10 th Avenue, Columbus, OH 43210, USA, Tel: (614) 688-7915; Fax: (614) 293-3465; E-mail: raphael.pollock@osumc.edu

\#These authors contributed equally.

Received date: September 24, 2016; Accepted date: October 17, 2016; Published date: October 24, 2016

Copyright: () 2016 Zewdu A, et al. This is an open-access article distributed under the terms of the Creative Commons Attribution License, which permits unrestricted use, distribution, and reproduction in any medium, provided the original author and source are credited.

\section{Abstract}

Objective: The heterogeneity of soft tissue sarcoma (STS) represents a major challenge for the development of effective therapeutics. Comprised of over 50 different histology subtypes of various etiologies, STS subsets are further characterized as either karyotypically simple or complex. Due to the number of genetic anomalies associated with genetically complex STS, development of therapies demonstrating potency against this STS cluster is especially challenging and yet greatly needed. Verticillin A is a small molecule natural product with demonstrated anticancer activity; however, the efficacy of this agent has never been evaluated in STS. Therefore, the goal of this study was to explore verticillin A as a potential STS therapeutic.

Methods: We performed survival (MTS) and clonogenic analyses to measure the impact of this agent on the viability and colony formation capability of karyotypically complex STS cell lines: malignant peripheral nerve sheath tumor (MPNST) and leiomyosarcoma (LMS). The in vitro effects of verticillin A on apoptosis were investigated through annexin V/PI flow cytometry analysis and by measuring fluorescently-labeled cleaved caspase 3/7 activity. The impact on cell cycle progression was assessed via cytometric measurement of propidium iodide intercalation. In vivo studies were performed using MPNST xenograft models. Tumors were processed and analyzed using immunohistochemistry (IHC) for verticillin A effects on growth (Ki67) and apoptosis (cleaved caspase 3).

Results: Treatment with verticillin A resulted in decreased STS growth and an increase in apoptotic levels after 24 h. $100 \mathrm{nM}$ verticillin A induced significant cellular growth abrogation after $24 \mathrm{~h}(96.7,88.7,72.7,57$, and 39.7\% reduction in LMS1, S462, ST88, SKLMS1, and MPNST724, respectively). We observed no arrest in cell cycle, elevated annexin, and a nearly two-fold increase in cleaved caspase 3/7 activity in all MPNST and LMS cell lines. Control normal human Schwann (HSC) and aortic smooth muscle (HASMC) cells displayed higher tolerance to verticillin A treatment compared to sarcoma cell lines, although toxicity was seen in HSC at the highest treatment dose. In vivo studies mirrored the in vitro results: by day 11, tumor size was significantly reduced in MPNST724 xenograft models with treatment of 0.25 and $0.5 \mathrm{mg} / \mathrm{kg}$ verticillin A. Additionally, IHC assessment of tumors demonstrated increased cleaved caspase 3 and decreased proliferation (Ki67) following treatment with verticillin A.

Conclusion: Advancement in the treatment of karyotypically complex STS is confounded by the high level of genetic abnormalities found in these diseases. Consequently, the identification and investigation of novel therapies is greatly needed. Our data suggest that verticillin A selectively inhibits MPNST and LMS growth via induction of apoptosis while exhibiting minimal to moderate effects on normal cells, pointing to verticillin $A$ as a potential treatment for MPNST and LMS, after additional preclinical validation.

Keywords: Verticillin A; Epipolythiodioxopiperazine alkaloid; Malignant peripheral nerve sheath tumor; MPNST; Leiomyosarcoma; LMS; Apoptosis; Soft tissue sarcoma; STS

\section{Introduction}

STS is a rare cancer of mesenchymal origin accounting for less than $1 \%$ of adult solid malignancies. This tumor cluster encompases over 50 subtypes, ranging from the highly metastatic (e.g., Ewing sarcoma) to those unable to metastasize from the primary site of development (e.g., 
desmoid tumor) [1]. These STS subsets are further characterized as genetically simple or complex cancers based on the presence or absence of fusion proteins as well as chromosome abnormalities [2,3]. Overall, STS patients experience high risks of local recurrence, distant metastasis, and unacceptably low disease-free survival [4-6], especially in the case of high grade STS. This reality points to the lack of effective systemic treatment options for patients. Conventional STS treatment includes chemotherapy and radiation therapy, as well as total surgical excision, all of which can have serious quality of life consequences [4]. Drug combinations, such as doxorubicin and ifosfamide, are commonly used in the treatment of these malignancies, albeit with modest efficacy:toxicity ratios [4-8].

Multiple chromosomal abnormalities including regional mutation, deletion, and amplification events render the effective treatment of complex karyotype STS extremely difficult [2,9]; the utilization of multiple aberrantly regulated pathways by these genetically complex malignancies not only complicates the development of targeted therapies but also increases the likelihood of therapeutic resistance. Identification of systemic therapy exhibiting selective potency with minimal patient toxicity is therefore critical.

Verticillin A is a small molecule member of a large and diverse family of epipolythiodioxopiperazine (ETP) alkaloids [10]. Identified as a fungal toxin released in response to pathogen infection, the anticancer capacity of verticillin A has been observed in metastatic colon carcinoma $[11,12]$. Although verticillin A displayed high efficacy as a single agent in this disease, combining verticillin A with TRAIL in a treatment approach resulted in both the potentiation of TRAIL as well as the reduction in the concentration of verticillin A required for demonstrable effect, hence our interest in evaluating the utility of this compound in STS.

\section{Materials and Methods}

\section{Reagents and drugs}

Verticillin A was purified from culture MSX59553 from the Mycosynthetix fungal library, as described in detail previously [10]; the sample was re-isolated from MSX59553 and was >95\% pure as measured by UPLC. Aliquots of verticillin A were reconstituted in dimethyl sulfoxide (DMSO; Fisher Bioreagents, Pittsburg, PA, USA) for in vitro studies or cremophor EL (Sigma-Aldrich, St. Louis, MO, USA) for in vivo investigations, and stored at $-20^{\circ}$ C. Antibodies (cleaved caspase 3, \#9661S and Ki67, "VP-K451) used for immunohistochemistry (IHC) assessment were purchased from Cell Signaling (Danvers, MA, USA) and Vector Laboratories (Burlingame, CA, USA), respectively, and used at a dilution of 1:1000. Propidium iodide (PI; ${ }^{\# P 4864-10 M L) ~ w a s ~ o b t a i n e d ~ f r o m ~ S i g m a-A l d r i c h ~(S t . ~ L o u i s, ~}$ MO, USA).

\section{Cell culture and cell lines}

Human NF1-associated MPNST cell lines, ST88 and S462, and sporadic MPNST cell line, MPNST724, have been previously described [13]. MPNST724 and ST88 were attained from Dr. Jonathan Fletcher (Brigham and Women's Hospital, Boston, MA, USA), and S462 was acquired from Dr. Lan Kluwe (University Hospital Eppendorf, Hamburg, Germany). LMS cell lines SKLMS1 and LMS1 were acquired from ATCC (Manassas, VA, USA) and Dr. Dominique Broccoli (Mercer University, Savannah, GA, USA), respectively. Cells were cultured in complete DMEM (DMEM with sodium pyruvate, L- glutamine, high glucose, 10\% FBS and $100 \mu \mathrm{g} / \mathrm{mL}$ Normacin) and maintained at $37^{\circ} \mathrm{C}$ at $5 \% \mathrm{CO}_{2}$ for the duration of the experiments. Human Schwann (HSC) and aortic smooth muscle (HASMC) cells (ScienCell, Carlsbad, CA, USA) were cultured in the appropriate media as described by supplier protocol and used as controls for in vitro assays. Primocin, used to supplement normal cell media, and Normacin, added to sarcoma cell media, were purchased from Invitrogen (Carlsbad, CA, USA). DMEM ( ${ }^{\# 11995-065)}$ was purchased from Thermo-Fisher (Grand Island, NY).

\section{Cell viability analysis}

MTS analysis was performed using CellTiter96 Aqueous NonRadioactive Cell Proliferation Assay kit (Promega, Madison, WI, USA) as specified by the manufacturer. Cells were seeded at a density of 5,000 cells per well, and allowed to adhere overnight. Cellular viability was assessed $24 \mathrm{~h}$ after treatment with DMSO (control), or 10, 50, or $100 \mathrm{nM}$ verticillin $\mathrm{A}$, and absorbance was measured at $490 \mathrm{~nm}$ wavelength.

\section{Clonogenic analysis}

Cells were seeded at 800 cells per well in a 6-well plate $\left(9.5 \mathrm{~cm}^{2}\right)$ and allowed to attach overnight prior to treatment with DMSO or varying concentrations of verticillin A. Cells were treated for $24 \mathrm{~h}$, then changed to recovery media and allowed to continue to grow for 10 days. Colonies were stained using $0.5 \%$ crystal violet solution in methanol for $30 \mathrm{~min}$. Staining solution was removed, wells were washed with deionized $\mathrm{H}_{2} \mathrm{O}$, and stained colonies were imaged and counted.

\section{Apoptosis analysis}

Apoptosis was measured by quantifying cleaved-caspase 3 and cleaved-caspase 7 activity using Cell Event Caspase 3/7 Green Detection Reagent (Life Technologies, Carlsbad, CA, USA) in the Incucyte Zoom system (Essen BioScience, Ann Arbor, MI, USA). Cells were plated at a density of 4,200 cells per well and allowed to adhere. The cells were then treated with DMSO or 10,50 , or $100 \mathrm{nM}$ verticillin A for $24 \mathrm{~h}$, and caspase activity was monitored using fluorescent Cell Event Caspase 3/7 Reagent (5 $\mu \mathrm{M}$ final concentration). Endpoint analysis was performed in each well using a final concentration of 10 $\mu \mathrm{M}$ Vybrant DyeCycle Green Stain (Life Technologies, Carlsbad, CA, USA) in complete DMEM. Apoptosis was confirmed using the Apoptosis Detection Kit (BD Pharmagen, San Diego, CA, USA) in complete DMEM. Per supplier instructions, $1 \times 106$ cells $/ \mathrm{mL}$ were stained with $5 \mu \mathrm{L}$ Annexin V-FITC and $5 \mu \mathrm{L}$ propidium iodide following $24 \mathrm{~h}$ treatment with DMSO or 10,50, or $100 \mathrm{nM}$ verticillin A and analyzed via FACS analysis (LSR II, BD Pharmagen, San Diego, CA, USA).

\section{Cell cycle analysis}

Cells were synchronized by $16 \mathrm{~h}$ starvation prior to treatment with DMSO, 10, 50, or $100 \mathrm{nM}$ of verticillin A for $24 \mathrm{~h}$. Attached and floating cells were collected following treatment and centrifuged for 5 min at 2500 RPM. Supernatant was removed and cells were washed twice with cold PBS. Cells were centrifuged (2500 RPM/5 min) and resuspended in $70 \%$ ethanol and fixed overnight at $-20^{\circ} \mathrm{C}$. Cells were then centrifuged at (2500 RPM/5 $\mathrm{min})$, and ethanol was removed. Subsequently, cells were resuspended in $400 \mu \mathrm{L}$ PI stain $(0.05 \mathrm{mg} / \mathrm{mL} /$ 
Citation: Zewdu A, Lopez G, Braggio D, Kenny C, Constantino D, et al. (2016) Verticillin A Inhibits Leiomyosarcoma and Malignant Peripheral Nerve Sheath Tumor Growth via Induction of Apoptosis. Clin Exp Pharmacol 6: 221. doi:10.4172/2161-1459.1000221

Page 3 of 7

$1 \mathrm{mg} / \mathrm{mL}$ sodium citrate/ $50 \mu \mathrm{L}$ Triton-X-100/ $50 \mu \mathrm{L}$ RNase A, brought to $50 \mathrm{~mL}$ with PBS) and analyzed via LSR II FACS analysis.

\section{In vivo animal models}

Six week old female SCID mice (Taconic Biosciences, Hudson, NY, USA; model ${ }^{\#}$ CB17SC) were injected subcutaneously with $1 \times 106$ MPNST724 cells into the flank. Once tumors reached $0.5 \mathrm{~cm}$, mice were allocated into three arms (10 mice per arm) and treatment via intraperitoneal injections was initiated: Vehicle (5\% ethanol/15\% cremophor EL/80\% PBS), verticillin A $(0.25 \mathrm{mg} / \mathrm{kg}$ every other day), or verticillin A $(0.5 \mathrm{mg} / \mathrm{kg}$ every other day). Mice were weighed and tumors were measured twice weekly. Mice were euthanized once tumors in the control group grew to $\sim 1.5 \mathrm{~cm}$. Final tumor volumes and weights were measured, and tumors were processed for IHC analysis.

\section{Immunohistochemistry and $\mathrm{H} \& \mathrm{E}$ analyses}

Ki67 (Vector Laboratories) and cleaved caspase 3 (Cell Signaling) antibodies were used to identify the effect of verticillin $\mathrm{A}$ on proliferation and apoptosis, respectively. IHC staining was conducted at Nationwide Children's Hospital (Columbus, $\mathrm{OH}$ ) and analysis was performed at the Polaris Innovation Center (The Ohio State University, Columbus, $\mathrm{OH}, \mathrm{USA}$ ). IHC samples and analyses were verified by pathologist, O. Hans Iwenofu, M.D. (The Ohio State University, Department of Pathology, Columbus, OH, USA).

\section{Statistics}

In vitro analyses were performed in triplicate. Mean \pm SEM (standard error mean) calculations and statistical analyses were performed for all cell-based assays, and EC50 values were computed using GraphPad Prism version 6.00 (for Windows, GraphPad Software, La Jolla California USA, www.graphpad.com). All Student $t$ tests performed were unpaired and two-sided. The average tumor volume $\left(\mathrm{mm}^{3}\right)$ and weight $(\mathrm{kg})$ for each study arm was measured and recorded. Mean \pm SEM was calculated for each treatment group, and end-point analyses determined using unpaired two-sided t test was utilized to determine variances. ${ }^{*} \mathrm{p} \leq 0.05 ;{ }^{* *} \mathrm{p} \leq 0.01 ;{ }^{* *} \mathrm{p} \leq 0.001$.

\section{Results}

\section{Verticillin A affects STS cellular viability and colony formation capability}

To assess the effect of verticillin A on STS, genetically complex MPNST and LMS cell lines were treated with increasing doses of verticillin A (Figure 1A). Primary cultured human Schwann (HSC) and smooth muscle (HASMC) cells were used to evaluate the effects of this compound on normal control cells. Verticillin A showed no effect on HASMC at $100 \mathrm{nM}$ and a displayed potency in HSC (EC50=64.94
nM) (Table 1) (Figure 1B). Following $24 \mathrm{~h}$ of treatment, verticillin A demonstrated a marked inhibitory effect on S462, ST88, and LMS1 at $10 \mathrm{nM}$ while MPNST724 and SKLMS1 exhibited tolerance at this dose (Figure 1C). All STS cell lines displayed a significant decrease in viability with $100 \mathrm{nM}$ verticillin $A$. The effects elicited in these complex karyotype STS cell lines, as compared to normal cells, suggest the possible selective inhibitory capacity of these agents.

Verticillin A also inhibited colony formation capacity in STS cells; with all doses of verticillin A, all MPNST and LMS cell lines were unable to form colonies (Figure 1D). We next investigated whether the response of these STS cells was due to a compound-mediated cell cycle arrest. No substantial arrest in cell cycle progression was demonstrable in MPNST or LMS (Figure 1E), indicating that anticancer effect of verticillin $\mathrm{A}$ is independent of cycle arrest (Figure 1).

\begin{tabular}{|l|l|}
\hline Cell Line & EC50 $(\mathbf{N m})$ \\
\hline HASMC & 250.7 \\
\hline HSC & 64.94 \\
\hline MPNST724 & 124.6 \\
\hline S462 & 26.03 \\
\hline SKLMS1 & 74.56 \\
\hline LMS1 & 10.69 \\
\hline
\end{tabular}

Table 1: EC50 values of normal and STS cell lines were determined via MTS assessment after $24 \mathrm{~h}$ treatment with verticillin A.

\section{Verticillin A induces apoptosis in MPNST and LMS}

Published works indicate that verticillin A impedes tumor growth via stimulation of apoptotic machinery [11]. Therefore, induction of apoptosis in STS was initially explored as a potential mechanism of action. Cleaved caspase 3 and 7 activities, markers of apoptosis, were measured using fluorescent Cell Event Caspase 3/7 Reagent. When activated, these caspases recognize the caspase-3/7 recognition motif (DEVD) on the Caspase 3/7 Reagent and cleave the substrate, thereby releasing the fluorescent DNA intercalating dye. The dye then binds DNA and produces a measurable signal. All STS cell lines displayed increased caspase activity whereas the effects on HSC and HASMC (1.1 and 1.2 fold change, respectively) were modest (Figure 2A). Annexin V/PI staining further supported this finding. All STS cell lines, but not normal control cells, exhibited significantly increased apoptosis upon verticillin A treatment (LMS1, $73.4 \pm 12.7 \%$; MPNST724, $34 \pm 4.3 \%$; SKLMS1, $29.4 \pm 2.3 \%$; S462, $25.9 \pm 3.75 \%$ ) (Figure 2B). Taken together, these data suggest that verticillin A inhibits STS growth via induction of apoptosis (Figure 2). 
Citation: Zewdu A, Lopez G, Braggio D, Kenny C, Constantino D, et al. (2016) Verticillin A Inhibits Leiomyosarcoma and Malignant Peripheral Nerve Sheath Tumor Growth via Induction of Apoptosis. Clin Exp Pharmacol 6: 221. doi:10.4172/2161-1459.1000221

A

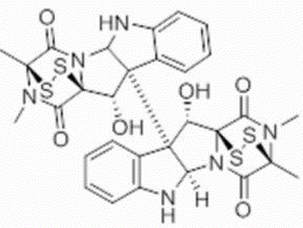

Verticillin A

B

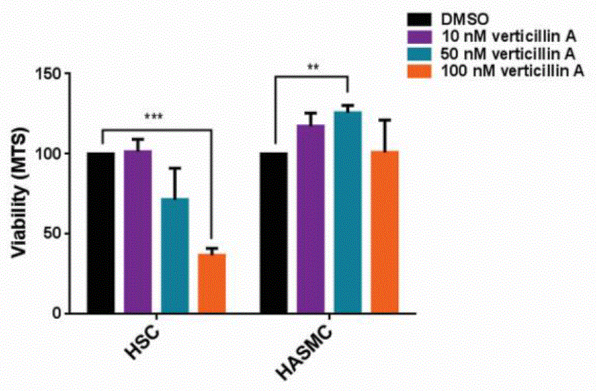

D

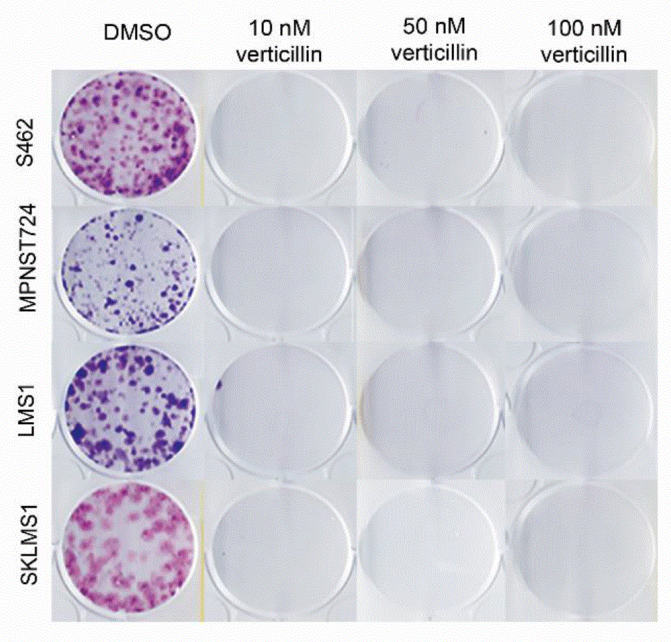

C

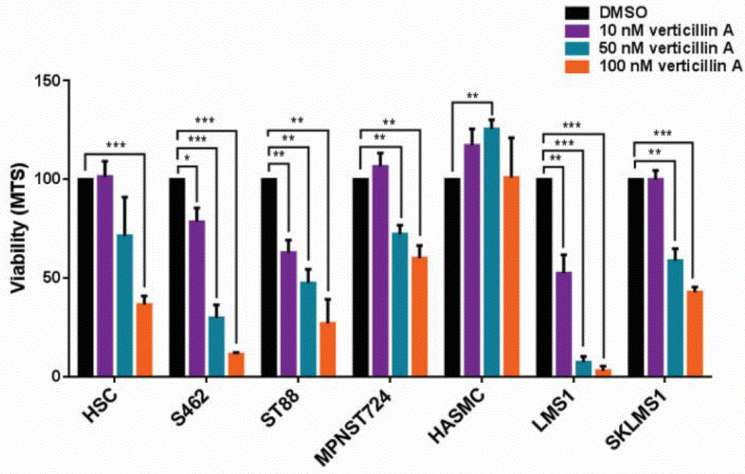

E

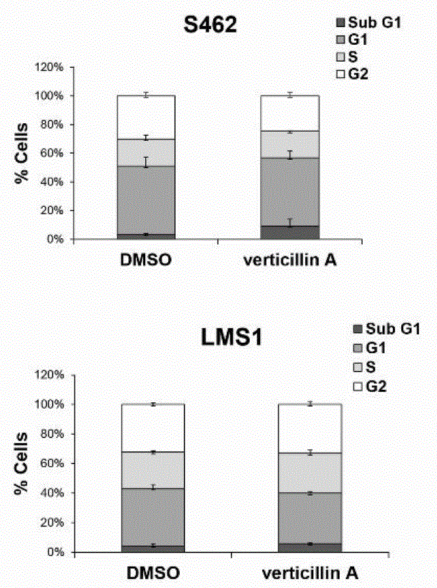

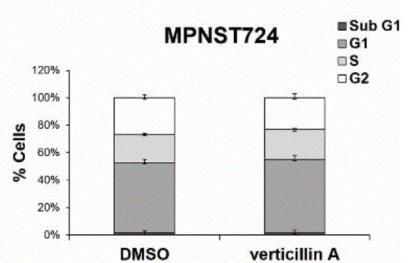

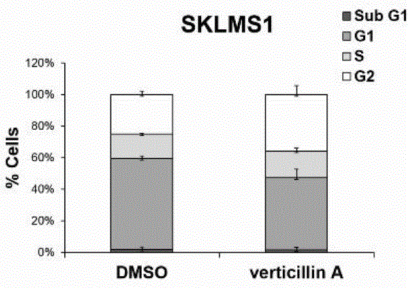

Figure 1: STS treated with verticllin A demonstrate decreased cell viability in vitro. (A) Molecular structure of verticillin A. (B) MTS analysis of normal cells (HASMC and HSC) treated with verticillin A at indicated doses for $24 \mathrm{~h}$. (C) MTS assessment of leiomysarcoma (SKLMS1 and LMS1) and MPNST (MPNST724 and S462) cell lines treated increasing concentrations of verticillin A for $24 \mathrm{~h}$. (D) Analysis of colony formation capability of S462, MPNST724, LMS1, and SKLMS1 upon treatment. (E) Cell cycle progression. ${ }^{*} \mathrm{p}<0.05,{ }^{* *} \mathrm{p}<0.01,{ }^{* * *} \mathrm{p}<0.001$. 
Citation: Zewdu A, Lopez G, Braggio D, Kenny C, Constantino D, et al. (2016) Verticillin A Inhibits Leiomyosarcoma and Malignant Peripheral Nerve Sheath Tumor Growth via Induction of Apoptosis. Clin Exp Pharmacol 6: 221. doi:10.4172/2161-1459.1000221

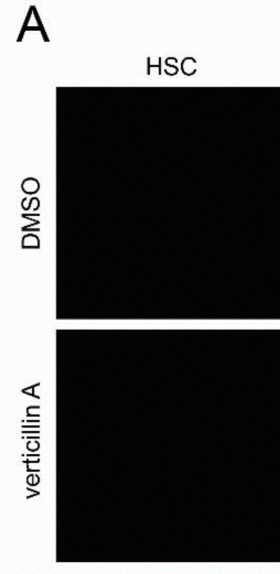

300 um

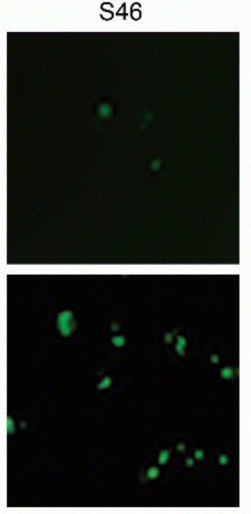

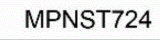

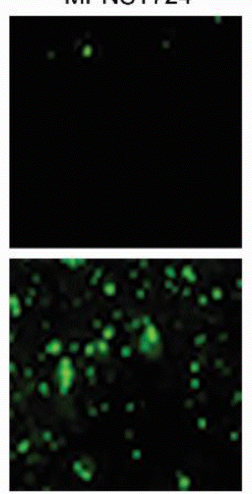

HASMC
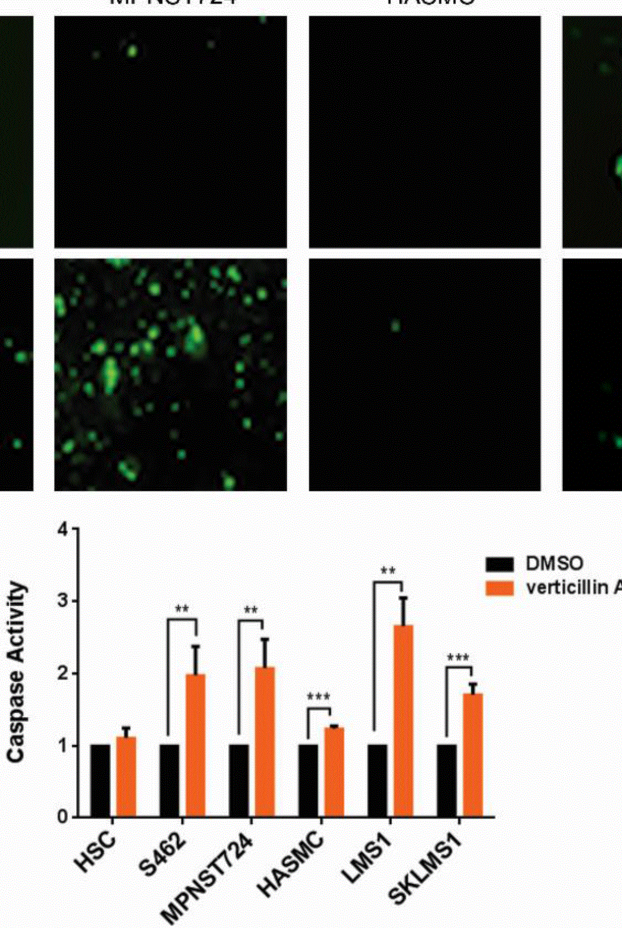

LMS1
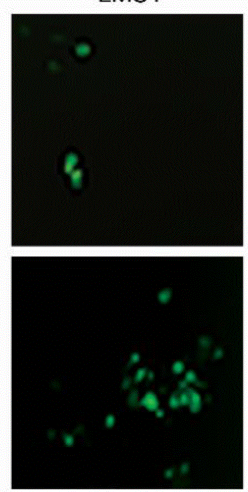

SKLMS1

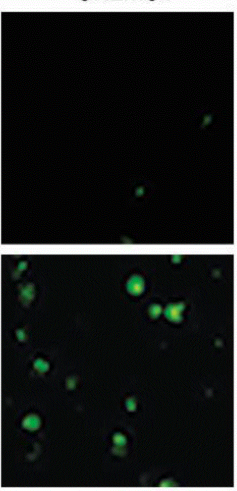

B
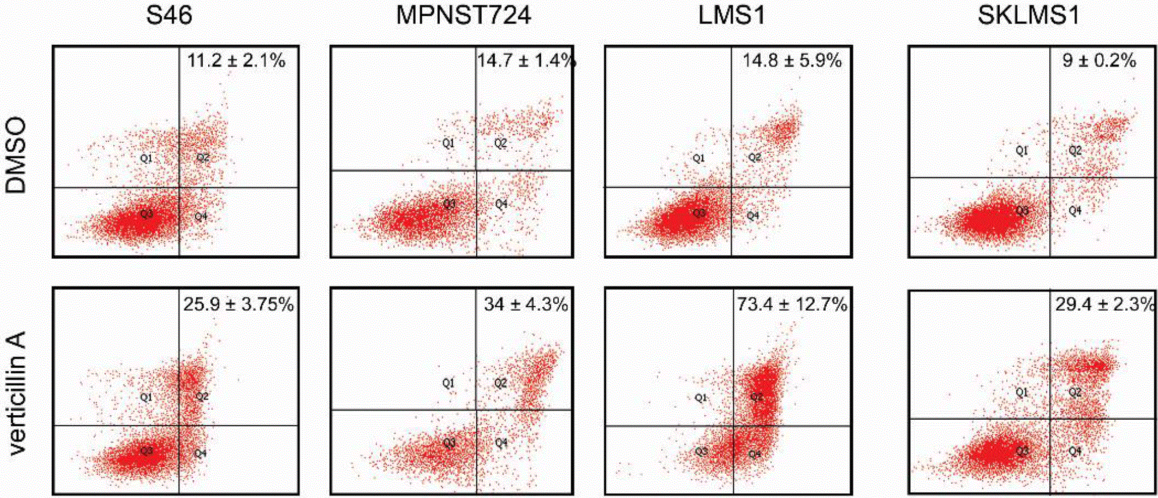

ANNEXIN V-FITC

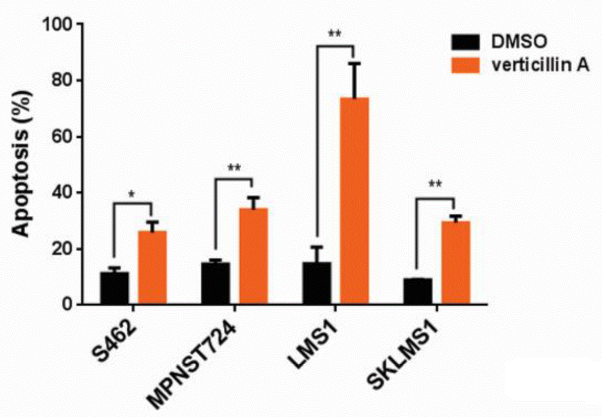

Figure 2: Verticillin A induces apoptosis in STS. (A) Cleaved caspase 3/7 activity as measured by time lapse microscopy. Representative images are shown. (B) Annexin V/PI FACS analysis of apoptotic levels. 
Citation: Zewdu A, Lopez G, Braggio D, Kenny C, Constantino D, et al. (2016) Verticillin A Inhibits Leiomyosarcoma and Malignant Peripheral Nerve Sheath Tumor Growth via Induction of Apoptosis. Clin Exp Pharmacol 6: 221. doi:10.4172/2161-1459.1000221

Page 6 of 7

\section{Verticillin A reduces tumor growth in vivo}

Next, the effects of verticillin A on STS tumor growth in vivo was investigated via use of MPNST724 xenografts. MPNST724 cells demonstrated higher tolerance to verticillin A (EC50=124.6 nM). It was therefore predicted that any efficacy seen in the MPNST724 xenograft models would be amplified in the other STS lines, as based on our EC50 data. MPNST724 xenografts demonstrated significant tumor growth inhibition in the mice allocated to either verticillin A treatment arms with the greatest effects observed with $0.5 \mathrm{mg} / \mathrm{kg}$ drug (tumor volume $=160.92 \mathrm{~mm}^{3}, \mathrm{p} \leq 4.05 \mathrm{E}-06$; tumor weight $=0.2 \mathrm{~g}, \mathrm{p} \leq$ 6.77E-05) (Figure 3A-C); treatment with $0.25 \mathrm{mg} / \mathrm{kg}$ also significantly reduced tumor volume $\left(355.89 \mathrm{~mm}^{3}, \mathrm{p} \leq 0.000147\right)$ by day 11 (Supplementary Figure 1).

Toxicity was observed in mice treated at the higher $0.5 \mathrm{mg} / \mathrm{kg}$ dose; mice demonstrated reduced grooming tendencies and mild weight loss (Figure 3D). Additionally, 2 mice were lost due to verticillin A-induced mortality. Further toxicology studies are therefore needed to understand the scope of these verticillin A-induced side effects.

Verticillin A also induced a nearly $40 \%$ decrease in the Ki67 proliferation marker expression in MPNST724 xenografts (Figure 3E). To identify compound-induced apoptosis in vivo, we probed for the cleaved caspase 3 apoptosis marker using IHC analysis. Verticillin A treatment led to a 5.5 -fold increase in cleaved caspase 3 expression in MPNST724 tumors (Figure 3D). Taken together, the observed in vitro selectivity and in vivo inhibitory effect of verticillin A supports further evaluation of its potential as a useful anti-STS clinical agent (Figure 3).

\section{Discussion}

Verticillin A is a small molecule with demonstrated anticancer activity via chromatin remodelling [10-12], although little is known on the effects of verticillin A on normal cells. Our data demonstrate the cytotoxic selectively of this agent toward STS cell lines compared to normal cells. While verticillin A impairs growth effects on these normal cells, their tolerance to verticillin A is greater than that of their STS counterparts in comparing HASMC to LMS. Likewise, Liu et al. demonstrated marked anticancer effects of verticillin A in colon carcinoma cell lines and higher tolerance in normal human colon epithelial cell (CCD-841) and donor $\mathrm{T}$ cells [11]. This selective responsiveness of cancer cells to verticillin A suggests the potential value of using these drugs in MPNST and LMS treatment.

Our data did not show cell cycle arrest in response to verticillin A; instead, verticillin A treatment resulted in an anticancer effect independent of cell cycle arrest. In contrast, verticillin A was shown to induce G2 cell cycle arrest in SW620 colon cancer cells, though no changes in cell cycle progression were observed in verticillin A-treated HepG2 liver carcinoma cells [11]. Taken together, these observations suggest that verticillin A may have various impacts on cell cycle progression depending on cell type being investigated, implying possible discrepancies in utilized pathways, differential metabolic processes, and differences in genetic and proteomic expression.

Minor signs of toxicity and compound-mediated mortalities were observed in the mice treated with the lower and higher doses of verticillin A, respectively. Further investigation is therefore needed to determine whether toxicity issues resolve following compound withdrawal. Toxicity levels may potentially be mitigated through a combinational therapeutic approach. In the exploration of combination TRAIL/verticillin A therapy on high grade colon carcinoma, Liu et al. were able to reduce the verticillin A dosage from 30-122 nM to $10 \mathrm{nM} 11$, thereby successfully sensitizing tumor cells to TRAIL treatment. Additionally, studies performed by our laboratory have demonstrated an effect of broad-spectrum histone deacetylase inhibitors (HDACis) on genetically complex STS [14,15], and improved efficacy:toxicity ratio through HDAC isoform-specific targeting [16]. Given the observed efficacy demonstrated by HDACis and verticillin A as single agents, investigation of combined HDACis/ verticillin $A$ therapeutic approaches may prove useful in the treatment of patients with high grade STS.

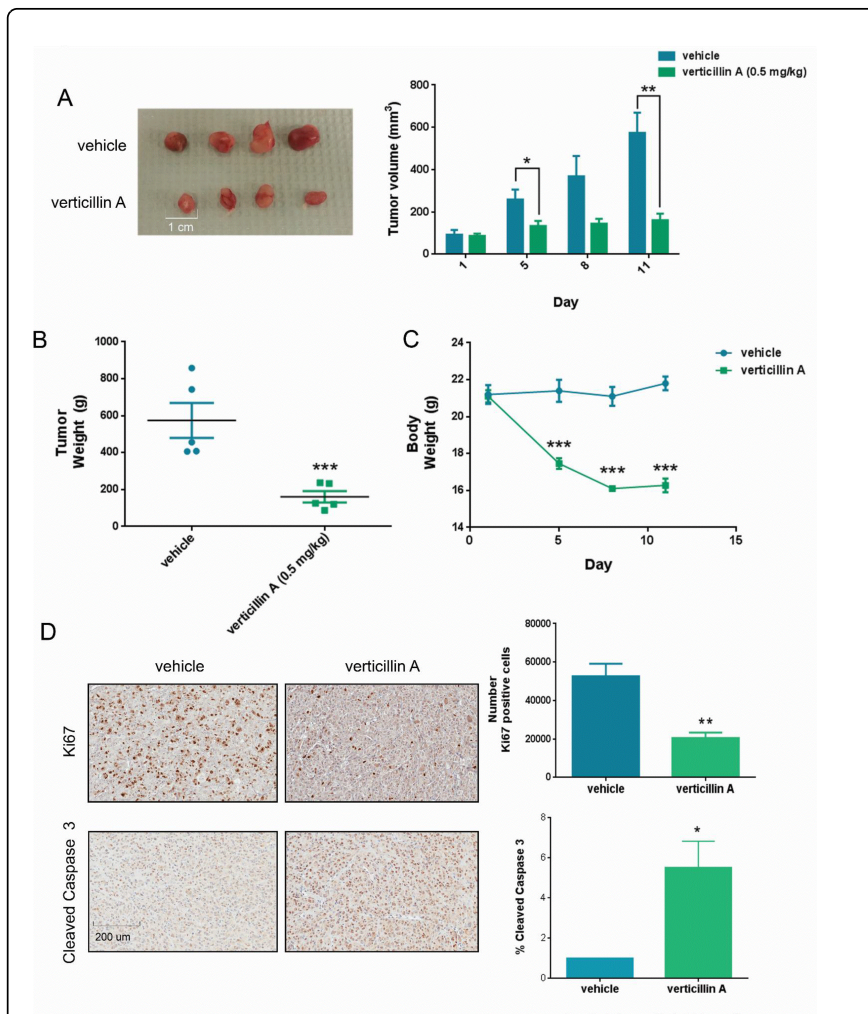

Figure 3: Verticillin A inhibits MPNST724 growth in vivo. (A and B.) Gross histology of tumors removed from mice treated with either vehicle or $0.5 \mathrm{mg} / \mathrm{kg}$ verticillin $\mathrm{A}$ at day 11. (B) Tumor burden by day $11(\mathrm{p}<0.0001)$. (C) Mouse body weight. (D) IHC analysis of proliferation (Ki67, $\mathrm{p}=0.015)$ and apoptosis (cleaved caspase $3, \mathrm{p}=0.042$ ) upon treatment with $0.5 \mathrm{mg} / \mathrm{kg}$ verticillin A treatment.

The study of the anti-tumor effects of natural compounds is a decades-long effort to identify more efficacious treatment strategies; between 1981 and 2010, 48.6\% of all new drugs introduced during this time were marine-, microorganism-, or plant-derived, roughly $65 \%$ of which are anticancer compounds $[17,18]$. These discoveries thereby support the continued investigation of new and understudied natural compounds in hopes of identifying novel compounds with selective anticancer potency.

Many natural compounds, such as romidepsin [19], have been shown to display growth inhibitory capacities in STS [20,21]. Given the success seen in the in vitro investigation of other bioactive compounds, investigation of the natural product verticillin A in STS was warranted. Our exploration of verticillin A suggests the possible value of this agent 
Citation: Zewdu A, Lopez G, Braggio D, Kenny C, Constantino D, et al. (2016) Verticillin A Inhibits Leiomyosarcoma and Malignant Peripheral Nerve Sheath Tumor Growth via Induction of Apoptosis. Clin Exp Pharmacol 6: 221. doi:10.4172/2161-1459.1000221

Page 7 of 7

in patient care; however, the results are still preliminary and will require further evaluation for the potential treatment of patients with MPNST and LMS.

\section{Acknowledgement and Funding}

The authors thank Dr. Kebin Liu, Dr. Amninder Kaur, David A. Kellough, Columbus Nationwide Children's Hospital, and the Polaris Innovation Center. This work was supported in part by the National Cancer Institute of the National Institutes of Health (U54CA168512 to R.E. Pollock and P01 CA125066 to N.H. Oberlies).

\section{References}

1. Matthyssens LE , Creytens D, Ceelen WP (2015) Retroperitoneal liposarcoma: current insights in diagnosis and treatment. Front Surg 2: 4.

2. Guillou L, Aurias A (2010) Soft tissue sarcomas with complex genomic profiles. Virchows Arch 456: 201-217.

3. Taylor BS, Barretina J, Maki RG, Antonescu CR, Singer S, et al. (2011) Advances in sarcoma genomics and new therapeutic targets. Nat Rev Cancer 11: 541-557.

4. Steen S, Stephenson G (2008) Current treatment of soft tissue sarcoma. Proc (Bayl Univ Med Cent) 21: 392-396.

5. Nakamura T, Grimer RJ, Carter SR, Tillman RM, Abudu A, et al. (2013) Outcome of soft-tissue sarcoma patients who were alive and event-free more than five years after initial treatment. Bone Joint J 95: 1139-1143.

6. Toulmonde M, Le Cesne A, Mendiboure J, Blay JY, Piperno-Neumann S, et al. (2014) Long-term recurrence of soft tissue sarcomas: prognostic factors and implications for prolonged follow-up. Cancer 120: 3003-3006.

7. Borden EC, Baker LH, Bell RS, Bramwell V, Demetri GD, et al. (2003) Soft Tissue Sarcomas of Adults: State of the Translational Science. Clin Cancer Res 9: 1941-1956.

8. Judson I, Verweij J, Gelderblom H, Hartmann JT, Schöffski P, et al. (2014) Doxorubicin alone versus intensified doxorubicin plus ifosfamide for first-line treatment of advanced or metastatic soft-tissue sarcoma: a randomised controlled phase 3 trial. Lancet Oncol 15: 415-423.

9. Post SM (2012) Mouse models of sarcomas: critical tools in our understanding of the pathobiology. Clin Sarcoma Res 2: 20.
10. Figueroa M, Graf TN, Ayers S, Adcock AF, Kroll DJ, et al. (2012) Cytotoxic epipolythiodioxopiperazine alkaloids from filamentous fungi of the Bionectriaceae. J Antibiot 65: 559-564.

11. Liu F, Liu Q, Yang D, Bollag WB, Robertson K ,et al. (2011) Verticillin A overcomes apoptosis resistance in human colon carcinoma through DNA methylation-dependent upregulation of BNIP3. Cancer Res 71: 6807-6816.

12. Paschall AV, Yang D, Lu C, Choi JH, Li X, et al. (2015) H3K9 Trimethylation Silences Fas Expression To Confer Colon Carcinoma Immune Escape and 5-Fluorouracil Chemoresistance. J Immunol 195: 1868-1882.

13. Lopez G, Torres K, Liu J, Hernandez B, Young E, et al. (2011) Autophagic survival in resistance to histone deacetylase inhibitors: novel strategies to treat malignant peripheral nerve sheath tumors. Cancer Res 71: 185-196.

14. Lopez G, Song Y, Lam R, Ruder D, Creighton CJ, et al. (2016) HDAC Inhibition for the Treatment of Epithelioid Sarcoma: Novel Cross Talk Between Epigenetic Components. Mol Cancer Res.

15. Lopez G, Liu J, Ren W, Wei W, Wang S, et al. (2009) Combining PCI-24781, a novel histone deacetylase inhibitor, with chemotherapy for the treatment of soft tissue sarcoma. Clin Cancer Res 15: 3472-3483.

16. Lopez G, Bill KL, Bid HK, Braggio D, Constantino D, et al. (2015) HDAC8, A Potential Therapeutic Target for the Treatment of Malignant Peripheral Nerve Sheath Tumors (MPNST). PLoS ONE.

17. Newman DJ, Cragg GM (2012) Natural products as sources of new drugs over the 30 years from 1981 to 2010. J Nat Prod 75: 311-335.

18. Newman DJ, Cragg GM (2016) Natural Products as Sources of New Drugs from 1981 to 2014. J Nat Prod 79: 629-661.

19. VanderMolen KM, McCulloch W, Pearce CJ, Oberlies NH (2011) Romidepsin (Istodax, NSC 630176, FR901228, FK228, depsipeptide): a natural product recently approved for cutaneous T-cell lymphoma. J Antibiot 64: 525-531.

20. Ree AH, Dueland S, Folkvord S, Hole KH, Seierstad T, et al. (2010) Vorinostat, a histone deacetylase inhibitor, combined with pelvic palliative radiotherapy for gastrointestinal carcinoma: the Pelvic Radiation and Vorinostat (PRAVO) phase 1 study. Lancet Oncol 11: 459-464.

21. Depsipeptide (Romidepsin) in Treating Patients With Metastatic or Unresectable Soft Tissue Sarcoma. ClinicalTrials.gov. 